\title{
Optical modeling of Ge-on-Si focal plane arrays with diffractive light coupling
}

\author{
D. Schmelz ${ }^{1}$, M. Steglich ${ }^{1}$, U. D. Zeitner ${ }^{1,2}$, E. B. Kley ${ }^{1}$ \\ ${ }^{1}$ Institute of Applied Physics, Abbe Center of Photonics, Friedrich Schiller University Jena, Albert- \\ Einstein-Straße 15, 07745 Jena, Germany \\ david.schmelz@uni-jena.de \\ ${ }^{2}$ Fraunhofer Institute for Applied Optics and Precision Engineering IOF, Albert-Einstein-Straße 7, \\ 07745 Jena, Germany
}

\begin{abstract}
:
Coupling light via stochastic needle-like nanostructures (black silicon) on the back side of a Ge-on-Si photodiode provides an increase of light absorption (and thus of the quantum efficiency) while preserving the $f_{3 \mathrm{~dB}}$-bandwidth of the diode [1,2]. When transferring this technique from a single photodiode to array-like devices such as focal plane arrays (FPAs), it comes to optical crosstalk in adjacent pixels.

A simulation model based on the ray tracing program Zemax can determine the absorption in Ge-onSi FPAs under arbitrary illumination. It simulates the absorption in every individual pixel and thus enables the calculation of optical crosstalk, as well as the total absorption, as functions of different design parameters. It gives the opportunity to compile parameter surveys or to perform a design optimization of the array device. The simulation model can be easily adapted to similar applications, thus providing a wide range of usage.
\end{abstract}

Key words: SWIR, Ge-on-Si, Focal Plane Array, Zemax, Simulation

\section{Introduction}

Ge-on-Si devices offer a promising potential alternative to the currently market leading III-Vsemiconductors for the detection of SWIR-light. They combine the SWIR-absorbing qualities of germanium with the largely established infrastructure of the silicon industry. A thin $\mathrm{Ge}$ layer enables the fabrication of detectors with a high $\mathrm{f}_{3 \mathrm{~dB}}$-bandwidth $[3,4]$ as well as the integration into CMOS manufacturing processes [5, 6].

Coupling the incoming light through the black silicon (b-Si) nanostructured device increases the quantum efficiency of a Ge-on-Si PIN diode by a factor of 3 to 4 depending on the wavelength [1]. Besides the antireflection (AR) effect of the $b$-Si, the increase of the quantum efficiency is caused by a broad transmissive scattering into the silicon (see Fig. 1) [7, 8]. Due to the high refractive index of silicon, it comes to total internal reflection inside the silicon and thus to a light trapping effect [2, 7, 8]. This extends the propagation length through the germanium and hence increases the absorption inside the PIN diode [2].
Whereas this technique works well for single diodes, in FPAs the scattering into high angles results in propagation and detection of the light rays at adjacent pixels which is known as "optical crosstalk". To reduce optical crosstalk, the substrate can be thinned out from the rear side and/or deep, thin trenches can be etched into the chip to separate the pixels from each other. In this way incoming light can be guided towards the designated pixels (see Fig. 2).

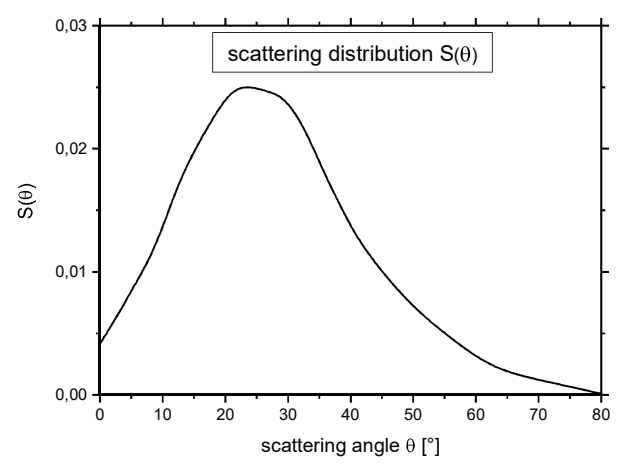

Fig. 1. Characteristic scattering distribution function of $b$-Si in transmission from air into silicon under normal incidence [7]. 
We present a method of simulating the light detection inside the Ge-on-Si array starting from the illumination up to the absorption within the photodiodes under different illuminances as well as variable design parameters such as pixel width, layer thickness, etc. This offers the possibility to investigate the influence of individual parameters and provides an efficient method to adapting the array device to specific detection requirements.

\section{Geometrical setup of the FPA}

Fig. 2 shows a schematic structure of the FPA. It consists of grid-like arranged, vertical PIN photodiodes on a Ge-on-Si chip with an epitaxial Ge layer of about $500 \mathrm{~nm}$ thickness. The base material silicon is, starting from a wavelength of $1200 \mathrm{~nm}$, nearly transparent which allows the guidance of the light through the $\mathrm{Si}$ towards the $\mathrm{Ge}$ layer without any losses. The b-Si nanostructures cover the back side of the chip and encompass the entire surface of the incidence window of the FPA. In order to achieve a more sophisticated but also more expensive device, one could also use a specific diffraction grating with an AR effect.

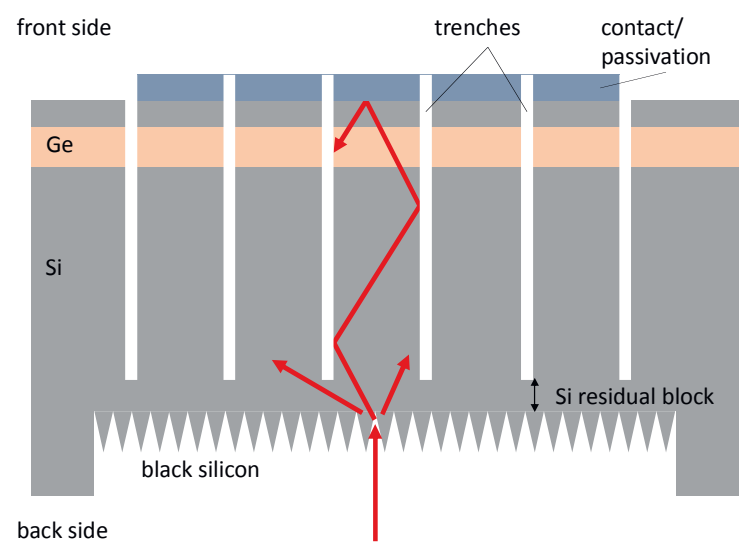

Fig. 2. Schematic structure of the Ge-on-Si FPA. The dimensions are not drawn to scale.

The epitaxial Ge layer consists of a thicker intrinsic layer which is covered by two highly doped $n$ - and p-layers. Due to high doping, the external quantum efficiency (EQE) in the PIN diode is the ratio of the intrinsic thickness, $d_{i}$, to the total thickness, $d_{t o t}$, multiplied by the total absorption, $A$, in the Ge layer [1]:

$$
E Q E=\frac{d_{i}}{d_{t o t}} \cdot A
$$

Thus, it is easy to conclude from the calculated absorption on the quantum efficiency. The front side is coated by a Si capping layer. On top of it $\mathrm{SiO}_{2}$ and Al layers serve for passivation and metalization of the photodiodes [3].
The trenches can be etched from either the front or the back side. Backfilling the trenches with $\mathrm{SiO}_{2}$ would possibly stabilize the chip. The high difference in refractive index between the trench and the silicon causes a waveguide effect where all light rays with a propagation angle below $73^{\circ}$ (for air), with respect to the surface normals, are directed to the Ge pixels. However, depending on the thickness of the $\mathrm{Si}$ residual block, the pixel length, the incidence angle or the position, a ray can also reach an adjacent pixel and generate optical crosstalk (see Fig. 2).

The whole geometric device can be modeled in the non-sequential mode of the ray tracing program Zemax which allows fast and variable simulations with good accuracy.

\section{Modeling of the b-Si nanostructure}

According to Füchsel et al. [9], all randomly arranged nanostructures with an aspect ratio larger than 1 can be named "black silicon". In this study, we designate b-Si as structures of stochastic, needle-like cones fabricated by a reactive ion etching process [8]. The results of a rigorous FDTD simulation of the reconstructed topography [7] were used to create the angular scattering distribution (see Fig. 1).

In Zemax, the angular scattering distribution function can be defined in a "BSDF" format (bidirectional scattering function). The fraction of the scattered intensity can be chosen. The residual intensity propagates in the direction of the specular ray. Furthermore, the values for reflectance and transmittance at the surface must be input into the program. This can be accomplished by a user-defined coating function which defines the transmittance and reflectance depending on the angle of incidence and the wavelength.

However, there is one problem which cannot be modeled straight forward: the values for transmission and reflection always apply to both propagation directions in Zemax. Although this rule is generally applicable for coatings, it is no longer valid for diffractive elements such as the b-Si structure. From air to silicon, it is almost perfectly transmissive, whereas vice versa it shows the already mentioned light trapping effect which means there is a great internal reflectance for light reaching the surface from the inside of the silicon (see Fig. 3). 


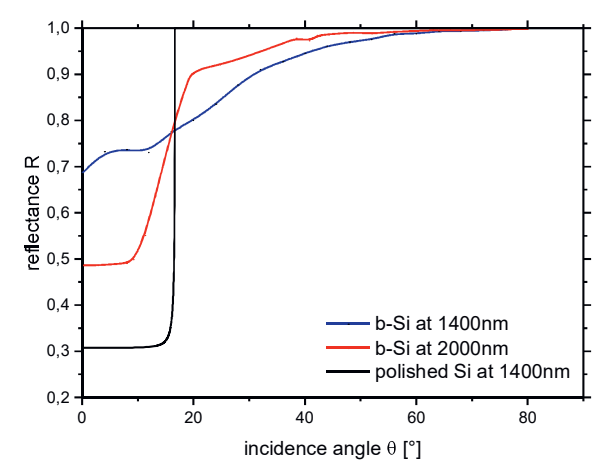

Fig. 3. Internal reflection of b-Si at the boundary from Si to air shown for wavelengths of $1400 \mathrm{~nm}$ and $2000 \mathrm{~nm}$. For comparison, the internal reflectance of polished Si is also plotted.

To work around this limitation, Zemax offers the use of a "source file". The incoming light is scattered and afterwards saved in a source file. The source file can be loaded into the actual Zemax model of the FPA. Within this model, the $\mathrm{b}-\mathrm{Si}$ is defined for its internal reflection (see Fig. 4).

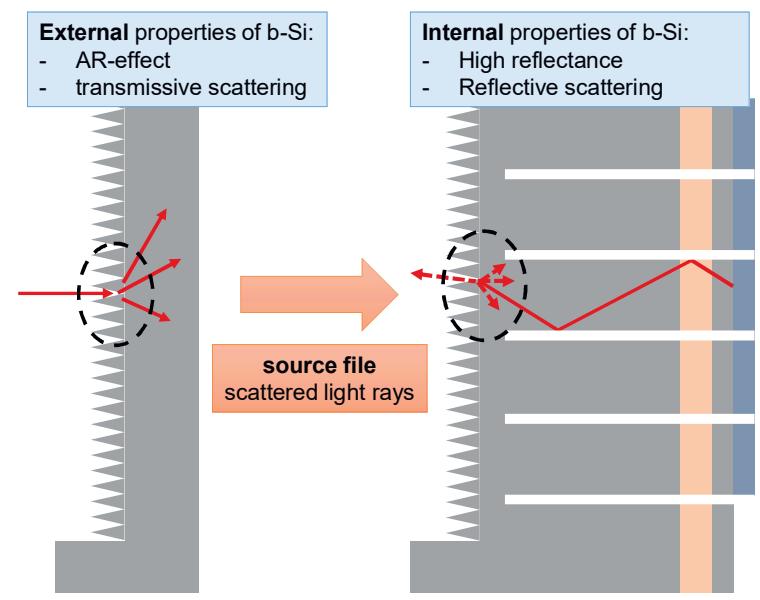

Fig. 4. Scheme of modeling the b-Si structure. In a separate Zemax object the transmissive scattering of $b$-Si from air to $\mathrm{Si}$ is simulated. The generated rays get saved in a source file. Subsequently the source file is loaded into the FPA model as a source object. Within the FPA model the internal characteristics of b-Si (see Fig. 3) are defined.

\section{Propagation inside the FPA model}

The rays that are loaded from the source file are traced through the simulation model. Every time a ray passes through the absorbing $\mathrm{Ge}$ layer, it loses some of its intensity according to the Lambert-Beer law. The rays are traced until their intensity reaches a minimum relative intensity of $10 \mathrm{E}-8$ compared to their initial intensity.

The "simple ray splitting" mode lowers the computation time significantly and enables a distinctly faster calculation. Instead of splitting a ray when it strikes a refractive boundary, it is either completely reflected or refracted. The decision of the trace is random but refers to the reflectance and transmittance as a probability. This requires a higher starting number of rays but keeps the number constant instead of increasing it exponentially.

To simulate the reflectance and transmittance of the coated front surface correctly, thin film interferences have to be taken into account. Zemax gives the opportunity to define a thin film coating of stacked layers from previously defined materials. The used coating algorithm considers the interference effects inside the layers. The validity of those Zemax calculations was confirmed using the open source software OpenFilters [10].

While the wave-optical consideration can be used for the thin capping layers, it cannot be applied to the $\mathrm{Ge}$ layer. Although Zemax considers the absorptance correctly, it does not submit the within a coating absorbed flux to the user. Hence, the absorption inside the germanium could not be measured in Zemax, if the $\mathrm{Ge}$ layer was included in the coating. Fortunately, because of the absorbing material and the broad angular illumination due to the scattering, the coherent approach can be neglected for the Ge layer. To prove the validity of the model, we used the fully coherent approach and calculated the results for reflection, transmission and absorption over the incidence angle with the help of the coating program Film Wizard (see Fig. 5). Scaling over the angular scatter distribution and then integrating gives approximately the same results as with a semi-coherent approach.

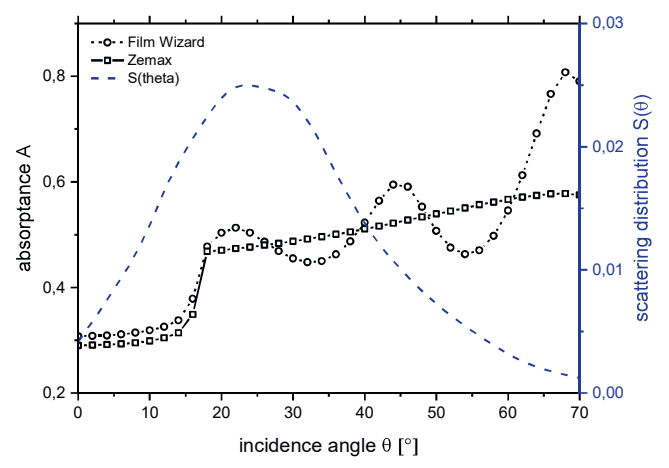

Fig. 5. Comparison between a completely coherent approach (Film Wizard, Ge and capping layers) and a semi-coherent approach (Zemax, only capping layers). Averaging the absorptance over the angular scattering distribution (blue) gives a deviation of less than $1.5 \%$.

Measurement of the absorbed irradiance

Zemax provides a pixelated object labeled as "Volume Detector". Its position and dimensions 
are chosen so that it spans the whole Ge layer. Thus, all of the light which is absorbed inside the $\mathrm{Ge}$ photodiodes can be detected. The resulting detected light can be depicted in the "detector viewer" of Zemax (see Fig. 6).
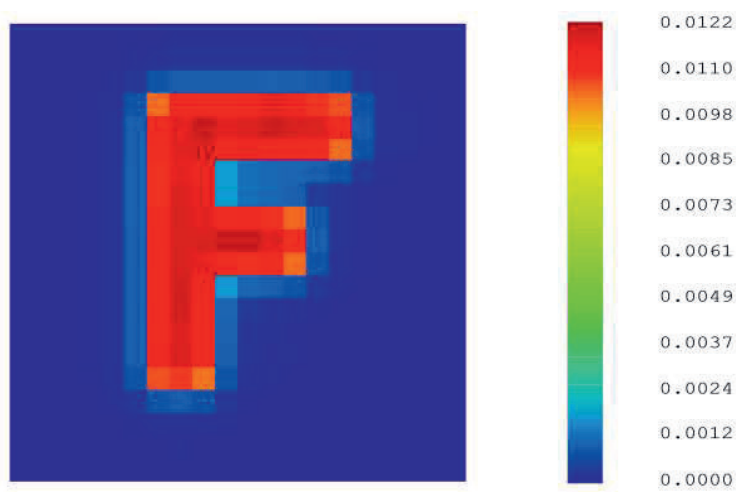

Fig. 6. Absorbed flux in the Ge layer of an imaged source in the shape of an " $F$ " under normal incidence. A single pixel represents one of the 20x20 PIN diodes. Design parameters: wavelength 1400 $\mathrm{nm}$, Ge layer thickness $500 \mathrm{~nm}$, pixel width $60 \mu \mathrm{m}$, Si residual thickness $5 \mu \mathrm{m}$.

Besides the absorption detector, the model is also framed by several "rectangular detectors" which measure the outgoing light. After a completed ray trace, the detectors can be read by a Python programming environment. It also enables an automatic modification of the design parameters of the model as well as a choice of the favored source file and the number of rays to trace. This allows an automatic creation of parameter studies which simplifies the evaluation of the model significantly.

\section{Simulation results}

Several influences from design parameters and illumination conditions were investigated in the implemented simulation model. To give an impression for the potential of the model, some of the simulation results are presented in this section.

Point spread functions (PSFs) offer a useful technique to study the amount of optical crosstalk with regards to certain parameters. For this purpose, a rectangular source illuminates the area of one pixel. The light which is detected in the designated pixel represents the desired intensity. The detected light in adjacent pixels represents the optical crosstalk. A typical PSF is shown in Fig 7.

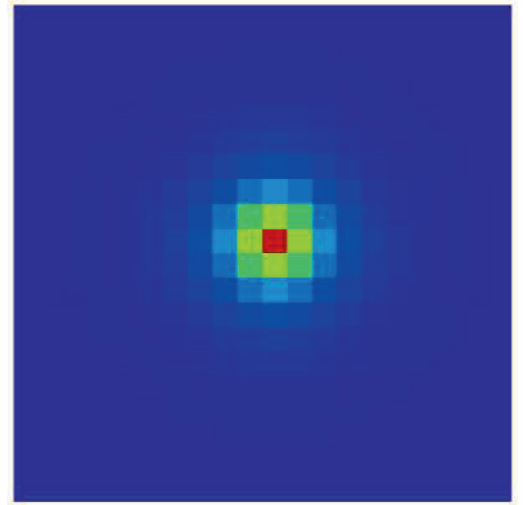

Fig. 7. Exemplary PSF of the absorbed flux detected in the Ge layer in Zemax at normal incidence. Design parameters: wavelength $1400 \mathrm{~nm}$, Ge layer thickness $500 \mathrm{~nm}$, pixel width $30 \mu \mathrm{m}$, Si residual thickness $50 \mu \mathrm{m}$.

Several investigations were performed by evaluating the PSFs under various parameter settings. In Fig. 8, the effect of the Si residual block thickness on the absorptance in the $\mathrm{Ge}$ layer can be regarded. It reveals the large influence the residual thickness has on the reduction of optical crosstalk. By increasing the residual thickness, the main pixel absorptance decreases exponentially, while simultaneously the crosstalk increases.

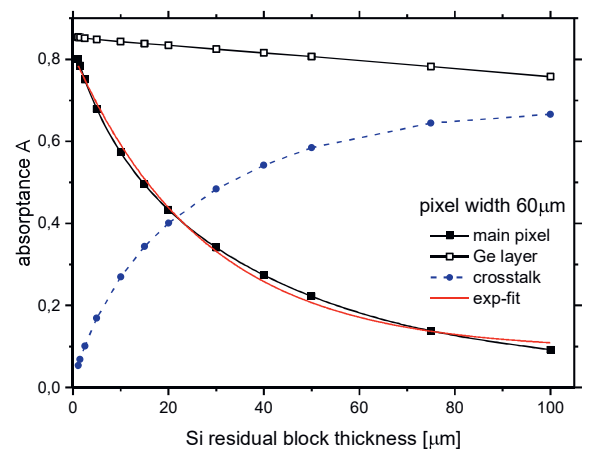

Fig. 8. PSF series about the absorbed flux depending on the thickness of the Si residual block at normal incidence. For better comparison, the total absorption in the $\mathrm{Ge}$ layer is also plotted. Design parameters: wavelength $1400 \mathrm{~nm}$, Ge layer thickness $500 \mathrm{~nm}$, pixel width $60 \mu \mathrm{m}$.

The high refractive index of silicon lowers the influence of the incidence angle because of the strong refraction towards the axis of incidence (see Fig. 9). This enables the detection of a homogeneous image even for large numerical apertures. The absorbed fluxes in the neighbor pixels above and below approximately compensate each other while the main pixel absorptance remains nearly constant. This implies that the crosstalk does not significantly increase with increasing field angle (i.e. from the image center to the outer areas). 


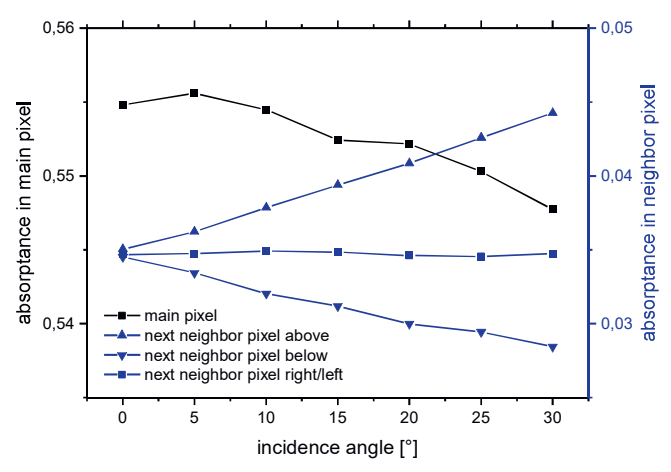

Fig. 9. PSF series about the absorbed flux depending on the incidence angle of the incoming light. Design parameters: wavelength $1400 \mathrm{~nm}$, Ge layer thickness $500 \mathrm{~nm}$, pixel width $30 \mu \mathrm{m}$, Si residual thickness $5 \mu \mathrm{m}$.

Knowing the PSF of an FPA also gives the opportunity of a deconvolution under certain conditions. This could provide a significant improvement to the detected image (see Fig. 10).

\section{References}

[1] M. Steglich, M. Oehme et al., Ge-on-Si photodiodes with black silicon boosted responsivity, Applied Physics Letters 107, 051103 (2015); doi: 10.1063/1.4927836

[2] M. Steglich, M. Zilk et al., Improvement of Ge-onSi photodiodes by black silicon light trapping, Applied Physics Letters 102, 111110 (2013); doi: 10.1063/1.4798322

[3] M. Oehme, J. Werner et al., High bandwidth Ge p-i-n photodetector integrated on Si, Applied Physics Letters 89, 071117 (2006); doi: $10.1063 / 1.2337003$

[4] G. Dehlinger, S. Koester et al., High-speed Germanium-on-SOI lateral PIN photodiodes, IEEE Photonics Technology Letters 16, 25472549 (2004); doi: 10.1109/LPT.2004.835631

[5] S. Koester, C. Schow et al., Ge-on-SOIdetector/Si-CMOS-amplifier receivers for highperformance optical-communication applications, Journal of Lightwave Technology 25, 46-57 (2007); doi: 10.1109/JLT.2006.888923

[6] K. Ang, T. Liow et al., Low thermal budget monolithic integration of evanescent-coupled Geon-SOI photodetector on Si CMOS platform, IEEE Journal on Selected Topics in Quantum Electronics 16, 106-113 (2010); doi: 10.1109/JSTQE.2009.2025142

[7] M. Kroll, T. Käsebier et al., Optical modeling of needle like silicon surfaces produced by an ICPRIE process, Proc. SPIE 7725, 772505 (2010); doi: $10.1117 / 12.854596$

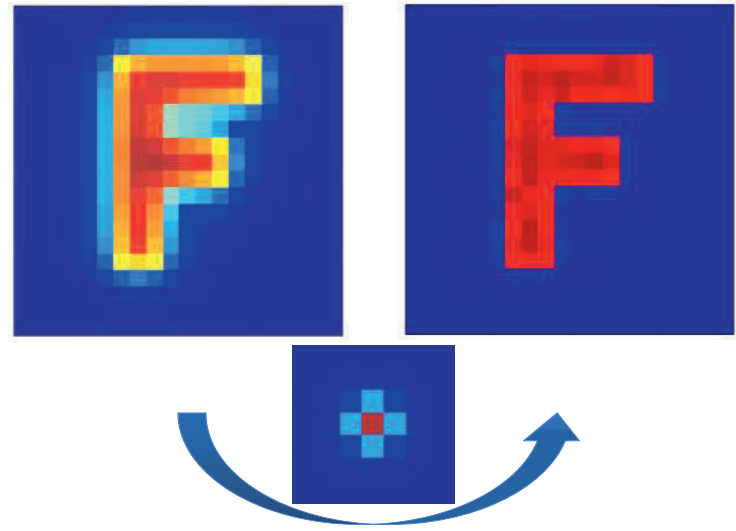

Fig. 10. Example of a deconvolution with the help of a determined PSF. This is yet only possible if the signal-to-noise ratio is sufficiently good.

\section{Acknowledgement}

This project is supported by the German Federal Ministry of Education and Research (BMBF), contract no. 03ZZ0435, in the context of the innovation alliance "3Dsensation".

[8] M. Steglich, T. Käsebier et al., The structural and optical properties of black silicon by inductively coupled plasma, Journal of Applied Physics 116, 173503 (2014);

doi: $10.1063 / 1.4900996$

[9] K. Füchsel, M. Kroll et al., Black Silicon Photovoltaics, Photon Management in Solar Cells, 117-151 (2015); doi: 10.1002/9783527665662.ch5

[10] S. Larouche, L. Martinu, OpenFilters: opensource software for the design, optimization, and synthesis of optical filters, Applied Optics 47, C219-C230 (2008); doi: 10.1364/AO.47.00C219 